\title{
Improvement of the Thermostability of Xylanase from Thermobacillus composti through Site-Directed Mutagenesis ${ }^{\mathbb{S}}$
}

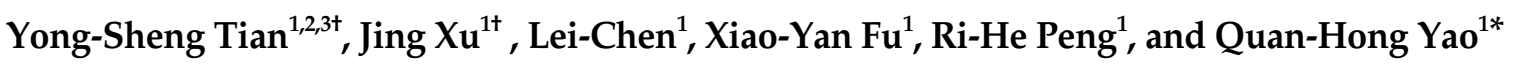 \\ ${ }^{1}$ Shanghai Key Laboratory of Agricultural Genetics and Breeding, Biotechnology Research Institute of Shanghai Academy of Agricultural \\ Sciences, Shanghai 201106, P.R. China \\ ${ }^{2}$ Shanghai Ruifeng Agricultural Science and Technology Co., Ltd, Shanghai 201106, P.R. China \\ ${ }^{3}$ College of Horticulture, Nanjing Agricultural University, Nanjing 210095, P.R. China
}

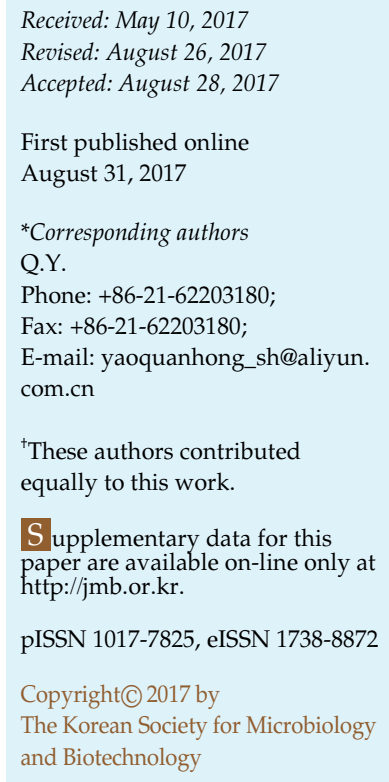

\begin{abstract}
Thermostability is an important property of xylanase because high temperature is required for its applications, such as wood pulp bleaching, baking, and animal feedstuff processing. In this study, XynB from Thermobacillus composti, a moderately thermophilic gram-negative bacterium, was modified via site-directed mutagenesis (based on its 3D structure) to obtain thermostable xylanase, and the properties of this enzyme were analyzed. Results revealed that the half-life of xylanase at $65^{\circ} \mathrm{C}$ increased from 10 to $50 \mathrm{~min}$ after a disulfide bridge was introduced between the $\alpha$-helix and its adjacent $\beta$-sheet at S98 and N145. Further mutation at the side of A153E named XynB-CE in the C-terminal of this $\alpha$-helix enhanced the half-life of xylanase for $60 \mathrm{~min}$ at $65^{\circ} \mathrm{C}$. Therefore, the mutant may be utilized for industrial applications.
\end{abstract}

Keywords: Xylanase thermostability, Thermobacillus composti, disulfide bridge, structure

\section{Introduction}

Xylans are the second-most abundant natural polysaccharides in cellulose and are considered renewable resources [1,2]. Xylan hydrolysis is an essential step for the degradation of lignocellulosic materials [3], and complete xylan degradation requires the concerted action of various glycosyl hydrolases (GHs). The most important $\mathrm{GH}$ is endo-1,4- $\beta$-xylanase (E.C. 3.2.1.8), which catalyzes the random hydrolysis of $\beta-1,4$-glycosidic bonds via a double displacement mechanism, thereby converting xylans to generate soluble xylooligosaccharides and xyloses [4]. According to amino acid sequence homologies and hydrophobic cluster analysis, xylanases have been grouped mainly into glycoside hydrolase families GH10 and GH11 [5, 6], and most of the industrially applied xylanases belong to
GH11 [7]. The structure of GH11 xylanases consists of two large $\beta$-sheets and one $\alpha$-helix, which form a structure resembling a partially closed right hand.

Xylanases are used in several high-temperature biotechnological processes, such as wood pulp biobleaching, baking, and animal feeding. However, these applications depend on the thermal stability of enzymes. Several thermostable families in GH11 xylanases have been cloned from thermophilic organisms to satisfy the demands of harsh conditions [8-13]. Another approach is to improve the thermal stability of xylanases by protein engineering to withstand extreme conditions.

The thermostability of xylanases has been largely enhanced by potential factors, such as charged amino acids, disulfide bridge introduction, and chimeric alterations near the $\mathrm{N}$ terminal region of a protein. Xylanase stability can be 
improved by introducing disulfide bridges in the $\mathrm{N}$-terminal of the protein [14-18].

In this work, the thermostability of Thermobacillus compost $i$ $\mathrm{XynB}$ was increased when a novel disulfide bridge was engineered into the $\mathrm{N}$-/C-terminal ends of the $\alpha$-helix of the protein. Our results suggested that the mutant may be used for industrial applications.

\section{Materials and Methods}

\section{Strain, Vector, and Chemicals}

Pichia pastoris strain GS-115 (His-Mut+) was purchased from Invitrogen, the expression vector pYPX88 (GenBank Accession No. AY178045) was prepared in our laboratory, and the pGEM-T Easy vector was obtained from Promega. Taq DNA polymerase, T4 DNA ligase, restriction endonucleases, and endoglycosidase $\mathrm{H}$ (Endo H) were procured from Takara and New England Biolab. Xylan from beechwood and xylose were purchased from Sigma. All other chemicals were of analytical grade.

\section{Synthesis and Modification of $X y_{n} B$}

On the basis of the amino acid sequence (GenBank Accession No. CP003255), we optimized XynB from T. composti by utilizing codon usage bias and tagged this gene with an additional C-terminal $6 \times$ His sequence. XynB was produced through a successive PCRbased two-step DNA synthesis method [19], and 14 primers were used (Table S1). The PCR was performed under the following conditions: $5 \mathrm{~min}$ at $94^{\circ} \mathrm{C}$, followed by 25 cycles of $20 \mathrm{sec}$ at $94^{\circ} \mathrm{C}$, $20 \mathrm{sec}$ at $58^{\circ} \mathrm{C}, 30 \mathrm{sec}$ at $72^{\circ} \mathrm{C}$, and a final extension at $72^{\circ} \mathrm{C}$ for $10 \mathrm{~min}$. The mutant genes $X y_{n} B-C$ and $X y n B-C E$ were obtained through site-directed mutagenesis [20]. The oligonucleotide sequences of $X y n B-C$ and $X y n B-C E$ are shown in Table S2.

\section{Expression of $X y n B$ Gene by P. pastoris}

The amplified fragment was digested with BamHI and SacI and then ligated into the eukaryotic expression vector pYPX88, which contains a $357 \mathrm{bp}$ fragment of $\alpha$-factor prepro-leader MF4I (GenBank Accession No. AY145833) with P. pastoris-preferred codon usage. MF4I is a substitute for a wild-type $\alpha$-signal sequence to enhance expression levels [21], and a standard recombinant method was used for routine DNA manipulations.

Recombinant pPIC9K-XynB was linearized with BglII to favor transformation into P. pastoris strain GS115 competent cells through electroporation. The $\mathrm{His}^{+}-\mathrm{Mut}^{+}$phenotype of the transformants was screened on a histidine-deficient Synthetic Dropout medium and incubated at $30^{\circ} \mathrm{C}$ for 3 days. Test expression experiments were performed to detect the expression of the target recombinant protein. The transformants with $\mathrm{His}^{+}$phenotype were streak cultivated on BMGY at $30^{\circ} \mathrm{C}$ for $24 \mathrm{~h}$. Two 96-well plates containing $60 \mu \mathrm{l}$ of BMMY medium with $1 \%(\mathrm{v} / \mathrm{v})$ methanol were incubated at $30^{\circ} \mathrm{C}$ for $12 \mathrm{~h}$. After $12 \mathrm{~h}$ of induction, positive colonies that contain xylanase activity were measured according to standard methods [22]. For large-scale protein production, the single isolated colony with a high expression level was cultured in $30 \mathrm{ml}$ of BMGY until the optical density at $600 \mathrm{~nm}$ reached 3.0; that is, P. pastoris reached log-phase growth. Subsequently, the cells were harvested and resuspended in an equal volume of BMMY medium at $30^{\circ} \mathrm{C}$ for 3 days. Xylanase expression was continuously induced by maintaining the concentration of methanol at $1 \%(\mathrm{v} / \mathrm{v})$ every $24 \mathrm{~h}$. Samples were taken at $0,24,48$, and $72 \mathrm{~h}$ to examine protein production through SDS-PAGE, and the xylanase activity was assayed concurrently.

\section{Protein Purification and Enzyme Assay}

The culture, which was induced with methanol for $72 \mathrm{~h}$, was harvested through centrifugation. Recombinant protein with additional six histidine residues at the C-terminal was purified using a $\mathrm{Ni}^{2+}$-NTA agarose affinity column (Sigma, China). The total protein concentration of the samples was determined via Bradford's dye-binding method, and bovine serum albumin was used as a standard [23]. The purified protein $(10 \mu \mathrm{g})$ was deglycosylated with $100 \mathrm{U}$ endoglycosidase $\mathrm{H}$ for $2 \mathrm{~h}$ at $37^{\circ} \mathrm{C}$. Deglycosylation production was conducted on $12 \%$ SDS-PAGE involving stacking and separating gels with $5 \%$ and $12 \%$ polyacrylamide, respectively. The spots of proteins after staining were visualized with Coomassie Brilliant Blue R-250.

Activity was assayed using $1 \%(\mathrm{w} / \mathrm{v})$ beechwood xylan in different buffers as substrates, and reducing sugars were determined with the dinitrosalicylic acid assay method [24, 25]. One unit of xylanase activity was defined as the amount of enzyme that catalyzed the formation of $1.0 \mu \mathrm{mol}$ reducing sugar equivalent to xylose from xylan per minute under the optimal assay conditions. All of the assays were carried out with an enzyme dilution appropriate for activity measurement. Each assay was repeated three times.

\section{Analysis of Kinetic Parameters for Xylanase}

The optimal $\mathrm{pH}$ was assayed in the following buffers: $\mathrm{Na}_{2} \mathrm{HPO}_{4}-$ citric acid buffer ( $\mathrm{pH}$ 2.0-7.0) and Tris- $\mathrm{HCl}$ buffer ( $\mathrm{pH} 7.0-10.0)$, at optimal temperature of $60^{\circ} \mathrm{C}$. $\mathrm{pH}$ stability was examined after incubation in buffers of $\mathrm{pH}$ varying from 2.0 to 10.0 at $37^{\circ} \mathrm{C}$ for $3 \mathrm{~h}$. The activity under standard reaction conditions $\left(60^{\circ} \mathrm{C}, \mathrm{pH} 6.0\right)$ without preincubation at different $\mathrm{pH}$ values was defined as $100 \%$.

The kinetic parameters $\left(K_{\mathrm{m}}\right)$ of xylanase activity were calculated from initial velocities at beechwood xylan concentrations ranging from 2 to $24 \mathrm{mg} / \mathrm{ml}$. The $K_{\mathrm{m}}$ values of xylanase were graphically determined by Lineweaver-Burk plotting.

\section{Analysis of Xylanase Thermostability}

The optimum temperature was determined at temperatures ranging from $20^{\circ} \mathrm{C}$ to $90^{\circ} \mathrm{C}$. Thermostability was obtained after preincubation in the absence of substrate for $30 \mathrm{~min}$ at varying temperatures. The half-life was measured at $65^{\circ} \mathrm{C}$ and $70^{\circ} \mathrm{C}$ for the preincubation of the enzyme solution for 10-60 min. Subsequently, the remaining activity was determined in each case. 


\section{Sequence Analysis and Homologous Modeling}

The deduced protein sequence was analyzed using the NCBI BLAST. Several xylanase amino acid sequences that belong to the published GH11 family were chosen for the alignment using DNAMAN. The active site of xylanase was identified on the basis of the alignment and known catalytic residues. The tertiary structural homology model and three-dimensional structures of xylanases were analyzed using SWISS-MODEL (http://swissmodel. expasy.org) and DSViewerPro60, respectively. Putative N-linked glycosylation sites were located with the NetNGlyc 1.0 program (http://www.cbs.dtu.dk/services/NetNGlyc/).

\section{Results}

\section{Homologous Alignment and Structural Assay}

Compared with other known characterization methods for xylanase, the amino acid sequence alignment of XynB indicated the presence of conserved residues at the active site. Some studies that have investigated the members of xylanase family $\mathrm{G}$ have demonstrated that the two conserved glutamate residues Glu76 and Glu169 can reach this cleft from opposite sides [22, 26, 27]. Aspergillus niger and T. compost $i$ xylanases were highly similar in three-dimensional structure (Fig. 1A), but their sequence identity was only $35 \%$. Crystallography studies on $A$. niger xylanase I have revealed that Glu79 and Glu170 are possibly the active nucleophile and the projected acid-base catalyst, respectively, and Gln129, Tyr70, Tyr81, and Asp37 residues may be involved in substrate binding [28]. Glu76 and Glu169 in XynB likely function as a nucleophile and an acid-base residue, respectively. The authenticity of the active-site glutamate residues Glu76 and Glu169 was identified using the PROSITE database (http://prosite.expasy.org). Gln124, Tyr67, Tyr78, and Asn33 were located in the XynB active site, and the stability of the catalytic site was maintained by hydrogen bond interactions. Asn33 is well correlated with optimum $\mathrm{pH}$ shifting [29], and N-bromosuccinimide, which is an amino acid modifier, restrains enzyme activity, thereby indicating the role of tryptophan residues in the catalytic function of xylanase [30].

\section{Design of Mutants}

The mutants of the $\mathrm{N}$ - and C-terminals of the $\alpha$-helix were constructed to investigate their effects on the thermal stability and other characteristics of XynB-WT. The mutation sites in the T. composti $\mathrm{XynB}$ structure are shown in Fig. 1C. Ser98 and Asn145 of the N-terminal were replaced with cysteine (S98C and N145C) and named XynB-C. Furthermore, Ala153 of the C-terminal of XynB-C was substituted with glutamic acid (S98C, N145C, and A153E) and labeled
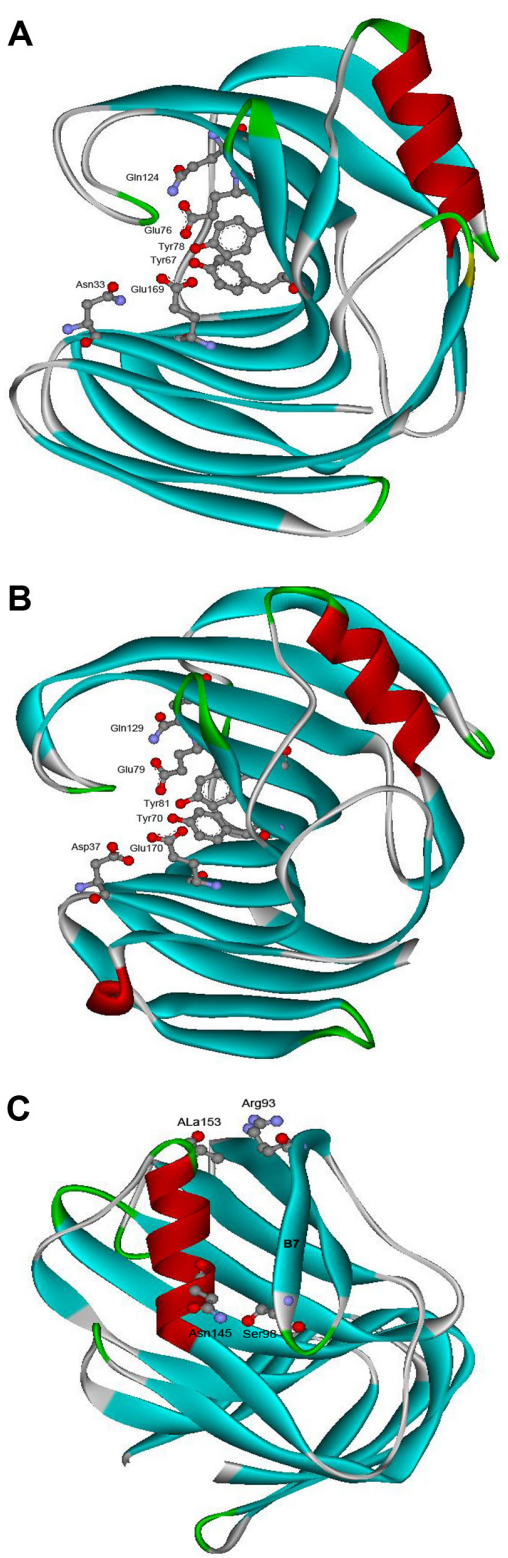

Fig. 1. Stereo-views of the structure of T. composti XynB (A) and xylanase I from Aspergillus niger (B), and (C) the location of stabilizing mutations in the T. compost $i$ XynB structure.

The $\alpha$-helix is shown in red, and $\beta$-sheets are in blue. The sites for the active site and mutations are indicated in black. The tertiary structural homology modeling and the three-dimensional structure of xylanases were analyzed by SWISS-MODEL (http://swissmodel.expasy.org) and DSViewerPro60.

\section{XynB-CE.}

The open reading frame of xylanase was $561 \mathrm{bp}$ in length, and it encoded a protein of 182 amino acids. SDS-PAGE revealed that $\mathrm{XynB}-\mathrm{WT}$ and its mutants had an apparent molecular mass of approximately $29 \mathrm{kDa}$. The size of 


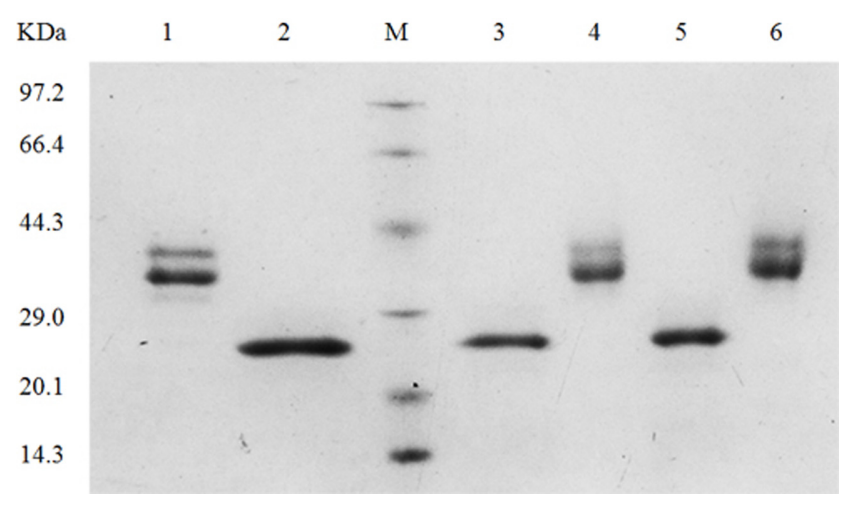

Fig. 2. SDS-PAGE patterns of the crude extract and deglycosylated xylanase.

Lane M: protein markers, Lane 1, 4, and 6: crude extract of xylanase; Lanes 2, 3 and 5: deglycosylation of xylanase with Endo-H. Lanes 1 and 2: XynB; Lanes 3 and 4: XynB-C; Lanes 5 and 6: XynB-CE.

xylanase molecules was theoretically $20 \mathrm{kDa}$ (Fig. 2), but native xylanase produced in $T$. composti was glycosylated under actual conditions. The possible glycosylation sites are shown in Fig. S1.

\section{Kinetic Parameters and Effect of $\mathrm{pH}$}

Enzyme activity as a function of $\mathrm{pH}$ was determined using $\mathrm{Na}_{2} \mathrm{HPO}_{4}$-citric acid buffer at $\mathrm{pH}$ 2.0-7.0 and Tris$\mathrm{HCl}$ buffer at $\mathrm{pH} 7.0-10.0$. The $\mathrm{pH}$ activity of the mutants was higher in the neutral-alkaline (7-10) regions than in Xyn-B-WT (Fig. 3A). The optimum $\mathrm{pH}$ values of the mutants and Xyn-B-WT were 8 and 6, respectively (Fig. 3A). The $\mathrm{pH}$ stability of xylanase was tested by incubating the enzyme solution in the absence of a substrate under diverse $\mathrm{pH}$ conditions for $3 \mathrm{~h}$ at $37^{\circ} \mathrm{C}$. XynB-CE was predominantly in the acidic region (5.5-6.5). However, XynB-C showed further stabilized activity in a wide $\mathrm{pH}$ range of 5-8 (Fig. 3B).

The $K_{\mathrm{m}}$ of XynB-WT was roughly $3.3 \mathrm{mg} / \mathrm{ml}$ (Table 1 and Fig. 2) when beechwood xylan was used as a substrate. The $K_{\mathrm{m}}$ of the mutant of XynB-C was similar to that of XynBWT. The $K_{\mathrm{m}}$ of XynB-CE was higher than those of XynB-C and XynB-WT. Moreover, the specific activities of XynBWT and XynB-C were 5,039 and 4,990 U/mg, respectively. However, the specific activity of XynB-CE $(3,993 \mathrm{U} / \mathrm{mg})$ was lower than those of XynB-WT and XynB-C. This finding indicated that $\mathrm{E} 153$ in combination with the disulfide bridge weakened the catalytic function.

\section{Temperature-Dependent Activity}

The resistance of XynB-WT, XynB-C, and XynB-CE to high-temperature inactivation was determined to investigate
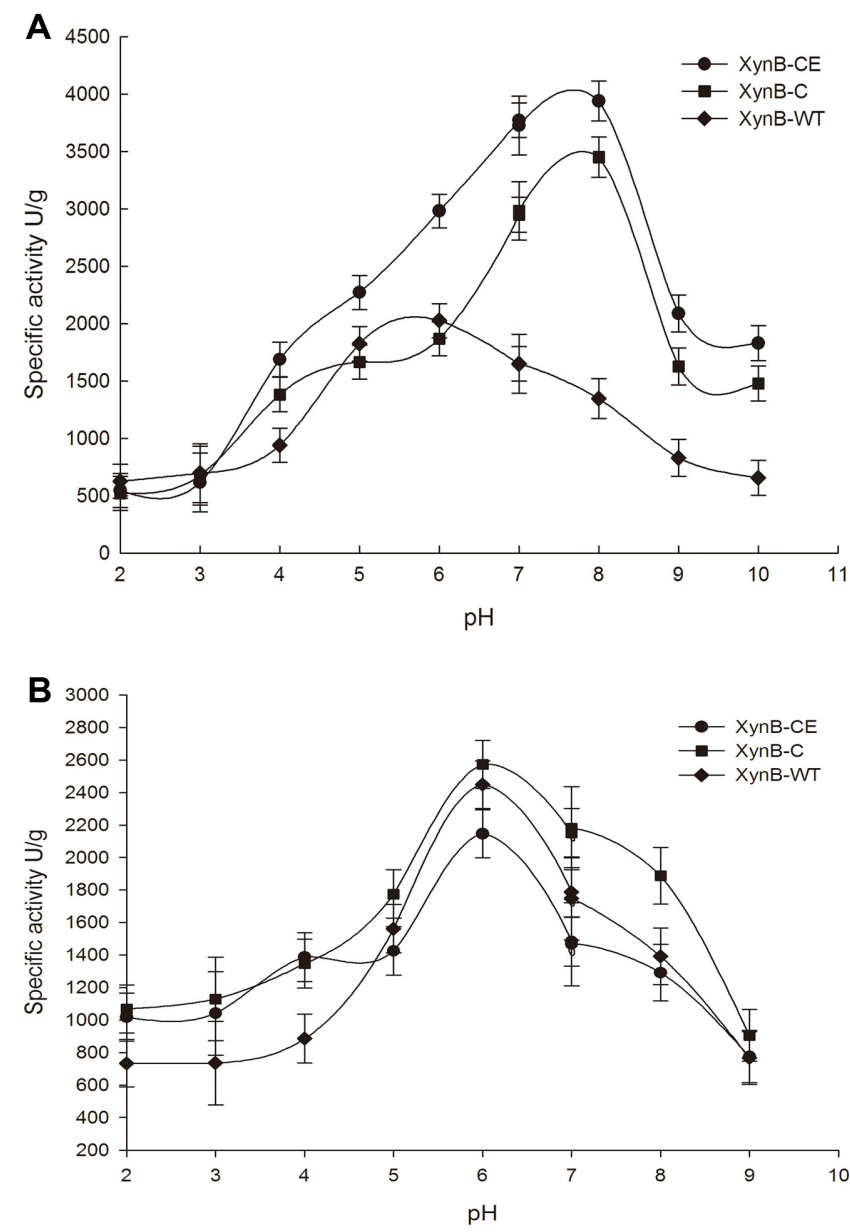

Fig. 3. Optimal $\mathrm{pH}(\mathbf{A})$ and $\mathrm{pH}$ stability (B) of the wild-type and mutant xylanases.

The optimal $\mathrm{pH}$ was assayed at various $\mathrm{pH}$ values $(2-10)$ at $60^{\circ} \mathrm{C}$. The $\mathrm{pH}$ stability, assayed at $37^{\circ} \mathrm{C}$ for $3 \mathrm{~h}$, was examined after incubation without substrate in buffers with $\mathrm{pH}$ varied from 2.0 to 10 .

Table 1. Half-lives $\left(T_{\text {half }}\right)$ at $65^{\circ} \mathrm{C}$ and $70^{\circ} \mathrm{C}$ and kinetic parameters of xylanases.

\begin{tabular}{lcccc}
\hline \multirow{2}{*}{ Enzyme } & \multicolumn{2}{c}{$T_{\text {half }}(\mathrm{min})$} & $K_{\mathrm{m}}(\mathrm{mg} / \mathrm{ml})$ & $\begin{array}{c}\text { Specific activity } \\
(\mathrm{U} / \mathrm{mg})\end{array}$ \\
\cline { 2 - 3 } & $65^{\circ} \mathrm{C}$ & $70^{\circ} \mathrm{C}$ & & 5,3 \\
\hline XynB & 10 & $\sim 4$ & 3.39 \\
XynB-C & 50 & $\sim 12$ & 3.5 & 4,990 \\
XynB-CE & $>60$ & $\sim 18$ & 13.3 & 3,993 \\
\hline
\end{tabular}

the contribution of the disulfide bond to thermostability. To compare the resistance of wild-type and mutant xylanases to inactivation at $65^{\circ} \mathrm{C}$ and $70^{\circ} \mathrm{C}$, we determined the half-lives $\left(T_{\text {half }}\right)$ of inactivation from the time-dependent inactivation curves. $T_{\text {half }}$ in mutations reached more than 60 min at $65^{\circ} \mathrm{C}$ compared with that of XynB-WT at $10 \mathrm{~min}$. At 

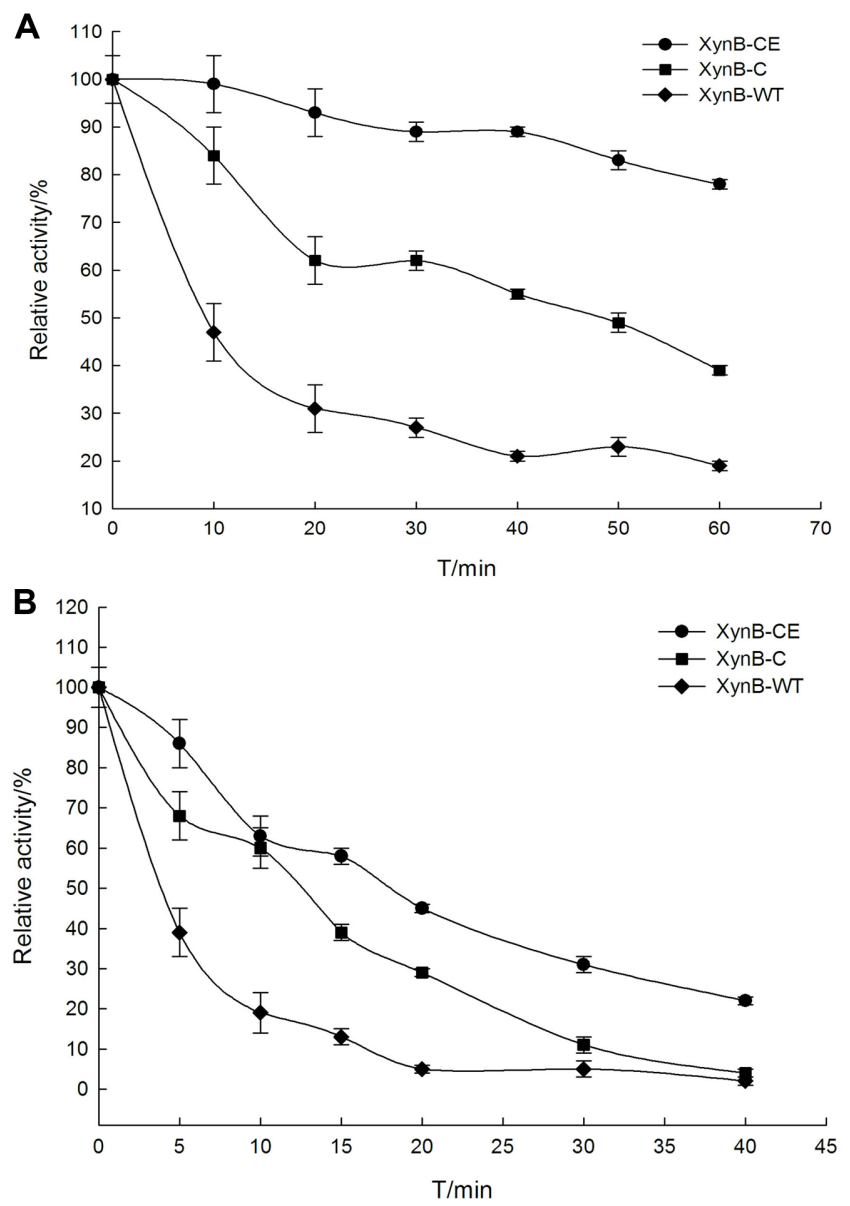

Fig. 4. Half-lives of wild-type $\mathrm{XynB}$ and mutants at $65^{\circ} \mathrm{C}(\mathbf{A})$ and $70^{\circ} \mathrm{C}(\mathbf{B})$.

The half-life was examined at the optimum $\mathrm{pH}$ after incubation without substrate.

$70^{\circ} \mathrm{C}, \mathrm{T}_{\text {half }}$ decreased to less than approximately $4 \mathrm{~min}$ in XynB-WT and roughly 12 and $18 \mathrm{~min}$ in the mutants (Table 1 ). XynB-C and XynB-CE that showed a 3-fold to 6-fold enhancement in $T_{\text {half }}$ indicated a stabilizing effect at $65^{\circ} \mathrm{C}$ and $70^{\circ} \mathrm{C}$ (Fig. 4).

To confirm the heat tolerance of mutant xylanase, we incubated the enzymes at various incremental temperatures in the absence of substrate for $30 \mathrm{~min}$ and measured the residual activity. Temperature-dependent inactivation curves suggested that the enzyme activity was more stable in the mutants than in the wild type (Fig. 5). The temperatures at which $50 \%$ of xylanase was inactivated for $30 \mathrm{~min}$ of incubation were $50^{\circ} \mathrm{C}$ and $60^{\circ} \mathrm{C}$ for the mutants. Conversely, the residual activity of $\mathrm{XynB}-\mathrm{WT}$ was only $40 \%$. At $20^{\circ} \mathrm{C}-$ $60^{\circ} \mathrm{C}$, the residual xylanase activity of $\mathrm{XynB}-\mathrm{WT}$ was between $30 \%$ and $40 \%$, whereas the average activity of the mutants

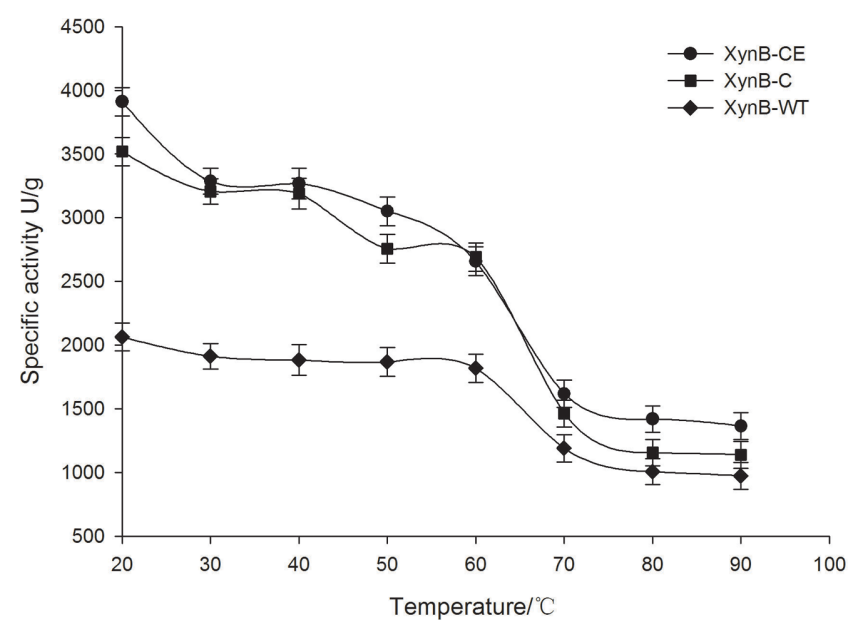

Fig. 5. Thermostability of the wild-type and mutant xylanases. The enzyme activity was measured at the optimum $\mathrm{pH}$ after $30 \mathrm{~min}$ incubation in the absence of substrate at different temperatures.

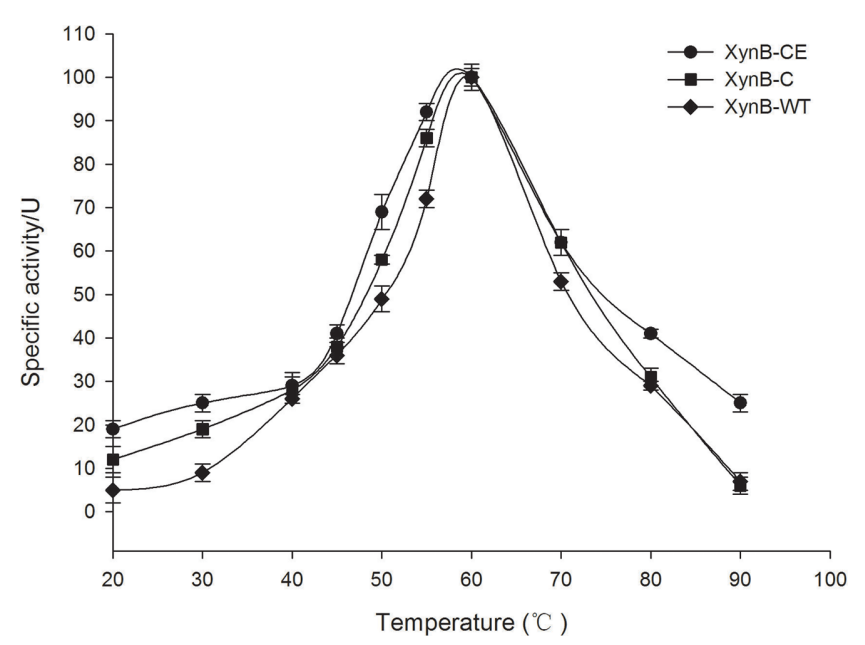

Fig. 6. Effect of temperature on the activity of wild-type and mutant xylanases.

Xylanase activity was determined at the optimum $\mathrm{pH}$ after $10 \mathrm{~min}$ pre-incubation in the presence of substrate at each temperature.

was more than $50 \%$. The residual activity of the mutants was enhanced at increased temperatures because of the increase in thermal resistance. The thermostability of XynB-CE was slightly higher than that of XynB-C because the combination of weakly stabilizing mutations (A153E) synergistically affected the thermostability of xylanase with a disulfide bridge (S98C-N145C). However, the optimal temperature of the mutants was similar to that of the wild type (Fig. 6). These results revealed that the disulfide bridge in the mutants greatly improves the relative activity of xylanase at the same temperature. 


\section{Discussion}

The use of xylanase in several industrial applications is dependent on the thermostability of the enzyme. Therefore, the thermostability of the enzyme was improved by protein engineering. For example, the thermostability of a Streptomyces xylanase was improved by introducing proline and glutamic acid residues [31]. The properties of the disulfide bridge are crucial factors for the increased thermostability of family 11 xylanases. A disulfide bridge introduced at the N-terminal in xylanase II enhanced thermostability [17, 26, 32-34].

Thermal stability can be increased by modifying a few residues in the primary sequence, possibly because the net free energy of the stabilization of native proteins is unexpectedly small [35]. New disulfide bonds inside proteins can be designed by comparing the xylanase II sequence from Trichoderma reese $i$ with the xylanase B sequence from T. composti, and this design shows that the presence of a disulfide bridge between these sequences corresponds to positions 98 and 145. Introducing a disulfide bond at the $\mathrm{N}$-terminal regions of the $\alpha$-helix improved the thermal stability of xylanases because the presence of a disulfide bond will restrict the motional freedom of the protein backbone in the unfolded state, thereby decreasing conformational entropy $[17,35,36]$. The combination of the mild stabilizing mutation with the disulfide bridge (S98C-N145C) elicits a cooperative effect on enzyme stability. In our study, the thermostability of $T$. compost $i \mathrm{XynB}$ was enhanced when the binding of the $\alpha$-helix (amino acids 143-152) to the protein core was improved at two separate sites ( $\mathrm{N}$ - and C-terminals of the $\alpha$-helix) compared with one site. A bulky side chain of the $\alpha$-helix in the C-terminal can possibly improve the packing of the nearby $\beta$-strand [17], and all of the amino acids with increased stability at positions 145 and 153 (cysteine, glutamic acid) can possibly improve the binding of the $\alpha$-helix to the nearby $\beta$-sheet B7 (amino acids 91-97, serine 98, arginine 93; Fig. 1B). The results of thermostability analysis indicated that XynB-CE is more stable than XynB-C. The C-terminal region of $\alpha$-helix Glu153 from the hydrogen bond over the cavity to the main chain $\beta$-sheet B7 (Arg93) may also play a role in the stabilization.

Although the $\alpha$-helix region strongly affects the thermostability of xylanase, excessive rigidity in this region may disturb the catalytic activity [17]. Certain factors are known to indicate a lower activity of XynB-CE: lower specific activity, limited $\mathrm{pH}$ stability ( $\mathrm{pH}$ 5.5-6.5), and higher $K_{\mathrm{m}}$. These findings suggest that the high rigidity of the $\alpha$-helix region decreases the catalytic activity of the enzyme, and the $\alpha$-helix region is correlated with thermal stability. Only introducing a disulfide bridge in $\mathrm{XynB}-\mathrm{C}$ does not evidently affect the catalytic activity, but this part increases the thermal stability of the enzyme. Hence, this finding provides the possibility of introducing other minor modifications reported to be critical factors that contribute to the thermophilic nature of family 11 xylanases. The optimum $\mathrm{pH}$ of the mutants increased to 8 , and the enzyme stability of XynB-C was higher than that of $\mathrm{XynB}-\mathrm{WT}$ (Fig. 3). No studies have reported changes in the optimum $\mathrm{pH}$ of a mutant with a disulfide bridge, and the mechanism is also unclear. This result provides a novel basis for investigations on the alkali resistance of xylanase.

In general, a disulfide bridge and minor mutations induce an additive increase in stability that is much greater than their individual effects. This experiment demonstrated that the introduction of disulfide bridges does not influence the expression of mutant xylanase. The strategic combination of disulfide bridges and minor mutations is suitable for the creation of xylanases for industrial applications.

\section{Acknowledgments}

This research was supported by the National Natural Science Foundation $(31672439 ; 31401458)$ and Key Project Fund of the Shanghai Municipal Committee of Agriculture (gongzi2014 7-1-3, zhongzi2016 1-2). The funders had no role in the study design, data collection and analysis, decision to publish, or preparation of the manuscript.

\section{References}

1. Collins T, Gerday C, Feller G. 2005. Xylanases, xylanase families and extremophilic xylanases. FEMS Microbiol. Rev. 29: 3-23.

2. Shi H, Zhang Y, Zhong H, Huang Y, Li X, Wang F. 2014. Cloning, over-expression and characterization of a thermotolerant xylanase from Thermotoga thermarum. Biotechnol. Lett. 36: 587-593.

3. Han HJ, Fu XY, Zhu B, Zhao W, Tian YS, Peng RH, et al. 2015. Characterization and high expression of recombinant Ustilago maydis xylanase in Pichia pastoris. Biotechnol. Lett. 37: 697-703.

4. Davies G, Henrissat B. 1995. Structures and mechanisms of glycosyl hydrolases. Structure 3: 853-859.

5. Jeffries TW. 1996. Biochemistry and genetics of microbial xylanases. Curr. Opin. Biotechnol. 7: 337-342.

6. Zhou C, Bai J, Deng S, Wang J, Zhu J, Wu M, et al. 2008. Cloning of a xylanase gene 44 from Aspergillus usamii and its expression in Escherichia coli. Bioresour. Technol. 99: 831-838.

7. Wang $\mathrm{Y}, \mathrm{Fu} \mathrm{Z}$, Huang $\mathrm{H}$, Zhang $\mathrm{H}$, Yao B, Xiong $\mathrm{H}$, et al. 2012. Improved thermal performance of Thermomyces 
lanuginosus GH11 xylanase by engineering of an N-terminal disulfide bridge. Bioresour. Technol. 112: 275-279.

8. Fukunaga N, Iwasaki Y, Kono S, Kita Y, Izumi Y. 1998. Thermostable xylanase. US Patent 5, 916,795.

9. Kumar PR, Eswaramoorthy S, Vithayathil PJ, Viswamitra MA. 2000. The tertiary structure at 1.59 A resolution and the proposed amino acid sequence of a family- 11 xylanase from the thermophilic fungus Paecilomyces varioti Bainier. J. Mol. Biol. 295: 581-593.

10. Morris DD, Gibbs MD, Chin CW, Koh MH, Wong KK, Allison RW, et al. 1998. Cloning of the $x y n B$ gene from Dictyoglomus thermophilum Rt46B.1 and action of the gene product on kraft pulp. Appl. Environ. Microbiol. 64: 1759-1765.

11. Paloheimo M, Mäntylä A, Vehmaanperä J, Hakola S, Lantto R, Lahtinen T, et al. 1998. Thermostable xylanases produced by recombinant Trichoderma reesei for pulp bleaching, pp. 255-264. In Claeyssen M, Nerinkx W, Piens K (eds.), Carbohydrate from Trichoderma reesei and Other Microorganisms. Royal Society of Chemistry, Cambridge, UK.

12. Samain E, Debeire P, Debeire-Gosselin M, Touzel JP. 1991. Xylanase, souches de Bacillus productrices de xylanase et leurs ytilisation. Patent FR-9101191.

13. Schlacher A, Holzmann K, Hayn M, Steiner W, Schwab H. 1996. Cloning and characterization of the gene for the thermostable xylanase XynA from Thermomyces lanuginosus. J. Biotechnol. 49: 211-218.

14. Fenel F, Leisola M, Janis J, Turunen O. 2004. A de novo designed N-terminal disulphide bridge stabilizes the Trichoderma reesei endo-1,4-beta-xylanase II. J. Biotechnol. 108: 137-143.

15. Jeong MY, Kim S, Yun CW, Choi YJ, Cho SG. 2007. Engineering a de novo internal disulfide bridge to improve the thermal stability of xylanase from Bacillus stearothermophilus No. 236. J Biotechnol. 127: 300-309.

16. Li YY, Zhong KX, Hu AH, Liu DN, Chen LZ, Xu SD. 2015. High-level expression and characterization of a thermostable xylanase mutant from Trichoderma reesei in Pichia pastoris. Protein Expr. Purif. 108: 90-96.

17. Turunen $\mathrm{O}$, Etuaho $\mathrm{K}$, Fenel F, Vehmaanpera J, Wu X, Rouvinen J, et al. 2001. A combination of weakly stabilizing mutations with a disulfide bridge in the alpha-helix region of Trichoderma reesei endo-1,4-beta-xylanase II increases the thermal stability through synergism. J. Biotechnol. 88: 37-46.

18. Song L, Dumon C, Siguier B, Andre I, Eneyskaya E, Kulminskaya A, et al. 2014. Impact of an N-terminal extension on the stability and activity of the GH11 xylanase from Thermobacillus xylanilyticus. J. Biotechnol. 174: 64-72.

19. Xiong AS, Yao QH, Peng RH, Li X, Fan HQ, Cheng ZM, et al. 2004. A simple, rapid, high-fidelity and cost-effective PCR-based two-step DNA synthesis method for long gene sequences. Nucleic Acids Res. 32: e98.

20. Peng RH, Xiong AS, Yao QH. 2006. A direct and efficient PAGE-mediated overlap extension PCR method for gene multiple-site mutagenesis. Appl. Microbiol. Biotechnol. 73:
234-240.

21. Xiong AS, Peng RH, Li X, Fan HQ, Yao QH, Guo MJ, et al. 2003. [Influence of signal peptide sequences on the expression of heterogeneous proteins in Pichia pastoris]. Sheng Wu Hua Xue Yu Sheng Wu Wu Li Xue Bao (Shanghai) 35: 154-160.

22. Miao S, Ziser L, Aebersold R, Withers SG. 1994. Identification of glutamic acid 78 as the active site nucleophile in Bacillus subtilis xylanase using electrospray tandem mass spectrometry. Biochemistry 33: 7027-7032.

23. Bradford MM. 1976. A rapid and sensitive method for the quantitation of microgram quantities of protein utilizing the principle of protein-dye binding. Anal. Biochem. 72: 248-254.

24. Miller GL Jr. 1959. Measurement of methods for assay of xylanase activity. Anal. Biochem. 2: 127-132.

25. Sun JY, Zhao D, Wang W, Liu J, Cheng J, Li Y, Jia YN. 2007. Expression of recombinant Thermomonospora fusca xylanase A in Pichia pastoris and xylooligosaccharides released from xylans by it. Food Chem. 104: 1055-1064.

26. Wakarchuk WW, Campbell RL, Sung WL, Davoodi J, Yaguchi M. 1994. Mutational and crystallographic analyses of the active site residues of the Bacillus circulans xylanase. Protein Sci. 3: 467-475.

27. Bray MR, Clarke AJ. 1994. Identification of a glutamate residue at the active site of xylanase A from Schizophyllum commune. Eur. J. Biochem. 219: 821-827.

28. Krengel U, Dijkstra BW. 1996. Three-dimensional structure of endo-1,4-beta-xylanase I from Aspergillus niger: molecular basis for its low pH optimum. J. Mol. Biol. 263: 70-78.

29. Qiu J, Han H, Sun B, Chen L, Yu C, Peng R, et al. 2016. Residue mutations of xylanase in Aspergillus kawachii alter its optimum $\mathrm{pH}$. Microbiol. Res. 182: 1-7.

30. Sapre MP, Jha H, Patil MB. 2005. Purification and characterization of a thermostable-cellulase free xylanase from Syncephalastrum racemosum Cohn. J. Gen. Appl. Microbiol. 51: 327-334.

31. Wang K, Luo H, Tian J. 2014. Thermostability improvement of a Streptomyces xylanase by introducing proline and glutamic acid residues. Appl. Environ. Microbiol. 80: 2158-2165.

32. Li H, Kankaanpää A, Xiong H. 2013. Thermostabilization of extremophilic Dictyoglomus thermophilum GH11 xylanase by an $\mathrm{N}$-terminal disulfide bridge and the effect of ionic liquid on the enzymatic performance. Enzyme Microb. Technol. 53: 414-419.

33. Yin X, Yao Y, Wu M C, 2014. A unique disulfide bridge of the thermophilic xylanase SyXyn11 plays a key role in its thermostability. Biochemistry (Mosc.) 79: 531-537.

34. Facchiano AM, Colonna G, Ragone R. 1998. Helix stabilizing factors and stabilization of thermophilic proteins: an X-ray based study. Protein Eng. 11: 753-760.

35. Davoodi J, Wakarchuk WW, Carey PR, Surewicz WK. 2007. Mechanism of stabilization of Bacillus circulans xylanase upon the introduction of disulfide bonds. Biophys. Chem. 125: 453-461.

36. Betz SF. 1993. Disulfide bonds and the stability of globular proteins. Protein Sci. 2: 1551-1558. 